\title{
Dynamin2 GTPase contributes to invadopodia formation in invasive bladder cancer cells
}

Yubai Zhang ${ }^{1}$, Maya Nolan ${ }^{1}$, Hiroshi Yamada $^{1}$, Masami Watanabe ${ }^{2}$, Yasutomo Nasu ${ }^{2}$, Kohji Takei $^{1, *}$ and Tetsuya Takeda ${ }^{1, *}$

${ }^{1}$ Department of Neuroscience, Okayama University Graduate School of Medicine, Dentistry and Pharmaceutical Sciences, Shikata-cho 2-5-1, Kita-ku, Okayama 700-8558, Japan

${ }^{2}$ Department of Urology, Okayama University Graduate School of Medicine, Dentistry and Pharmaceutical Sciences, Shikata-cho 2-5-1, Kita-ku, Okayama 700-8558, Japan

* Corresponding authors

Dr. Tetsuya Takeda, Department of Neuroscience, Okayama University Graduate School of Medicine, Dentistry and Pharmaceutical Science, 2-5-1 Shikata-cho, Kita-ku, Okayama 7008558, Japan

Tel: (+81)-86-235-7120; Fax: (+81)-86-235-7126; email: ttakeda@ okayama-u.ac.jp

Dr. Kohji Takei, Department of Neuroscience, Okayama University Graduate School of Medicine, Dentistry and Pharmaceutical Science, 2-5-1 Shikata-cho, Kita-ku, Okayama 7008558, Japan

Tel: (+81)-86-235-7121; Fax: (+81)-86-235-7126; email: kohji@ md.okayama-u.ac.jp 


\section{Abstract}

Cancer cell invasion is mediated by actin-based membrane protrusions termed invadopodia. Invadopodia consist of "core" F-actin bundles associated with adhesive and proteolytic machineries promoting cell invasion by degrading extracellular matrix (ECM). Formation of the F-actin core in invadopodia is regulated by various actin-binding proteins including Arp2/3 complex and cortactin. Dynamin GTPase localizes to the invadopodia and is implicated in cancer cell invasion, but its precise role at the invadopodia remained elusive.

In this study, we examined the roles of dynamin at the invadopodia of bladder cancer cells. Although all three dynamin isoforms (dynamin1, 2 and 3) are expressed in human bladder cancer cell line T24, only dynamin2 localizes to the invadopodia. Inhibition of dynamin2 function, using either RNA interference (RNAi) or the dynamin specific inhibitor Dynasore, caused defects in invadopodia formation and suppressed invasive activity of T24 bladder cancer cells. Structure-function analysis using dynamin2 deletion fragments identified the proline/arginine-rich domain (PRD) of dynamin2 as indispensable for invadopodia formation and invasiveness of T24 cells. Thus, dynamin2 contributes to bladder cancer invasion by controlling invadopodia formation in bladder cancer cells and may prove a valuable therapeutic target.

Keywords: dynamin, invadopodia, bladder cancer, cortactin, actin 


\section{Abbreviations}

Arp2/3: actin related protein 2 and 3

WASP: Wiskott-Aldrich Syndrome protein

WIP: WASP-interacting protein

MMP2/9: matrix metalloprotease 2 and 9

MT1-MMP: membrane type-I matrix metalloprotease

SV40: simian virus 40

HRP: horseradish peroxidase

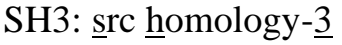




\section{Introduction}

Bladder cancer is the second most common urological cancer worldwide with a $70 \%$ recurrence rate after surgery of superficial (non-muscle invasive ) stage [1, 2]. Radical cystectomy (RC) remains the primary option for the muscle-invasive bladder cancer where it cures approximately $2 / 3$ of patients [3]. Despite this, quality of life of patients and their caregivers is significantly affected after RC and social healthcare system suffers from this heavy burden [4]. Thus alternative approaches efficiently restricting bladder cancer within its original local lesion for subsequent non-radical treatments need to be developed.

Invadopodia are adhesive membrane protrusions formed at the ventral surface of metastatic cancer cells including bladder cancer [5, 6] . Invadopodia exhibit three core neoplastic functions associated with malignancy; invasiveness (requiring F-actin, Arp2/3, WASP/WIP, cortactin), adhesion (requiring $\alpha / \beta$ integrins) and proteolysis (requiring MT1-MMP and MMP2/9) [7]. Besides basement membrane penetration, invadopodia also participate in essential steps of lethal distant metastasis, stromal invasion, intravasation and extravasation [8]. Thus inhibition of invadopodia formation and/or function has promise for the therapeutic suppression of cancer cell invasion and metastasis [9].

Dynamin is a large GTPase essential for membrane fission in clathrin-mediated endocytosis [10]. There are three dynamin isoforms in mammalian cells: dynamin1 (neuronal isoform), dynamin2 (ubiquitous isoform) and dynamin3 (a testis, lung and brain isoform) [10]. All the dynamin isoforms share similar domain structures aligned from $\mathrm{N}$-terminus to $\mathrm{C}$-terminus: a GTPase domain, a middle domain, a PH domain, a GTPase effector domain (GED) and PRD 
domain [10]. Dynamin also regulates the actin cytoskeleton either directly [11] or indirectly via interaction with various actin-binding proteins such as cortactin [12]. Dynamin2 has been implicated in invadopodia function in highly invasive melanoma cells [13] and lung cancer cells [14], but its precise roles are yet unknown. Here we report for the first time that dynamin 2 contributes to invasiveness of bladder cancer cells by regulating invadopodia formation. 


\section{Materials and Methods}

\subsection{Cell culture}

SV40 immortalized transitional epithelium cell line SV-HUC-1 (ATCC No. CRL-9520) was obtained from RIKEN BRC and transitional cell carcinoma T24 (ATCC No. HTB-4) was obtained from ATCC. Cells were cultured in RPMI-1640 medium (Wako Pure Chemical Industries) supplemented with $10 \%$ fetal bovine serum and $1 \%$ penicillin/streptomycin at $37^{\circ} \mathrm{C}$ in humidified air with $5 \% \mathrm{CO}_{2}$.

\subsection{Reagents}

Monoclonal rabbit anti-dynamin 1 antibody (ab52611) was purchased from Abcam, polyclonal goat anti-dynamin 2 antibody (sc-6400) was from Santa Cruz, polyclonal rabbit antidynamin 3 antibody (PA1-662) was purchased from Thermo Fisher Scientific, monoclonal mouse anti- $\beta$-Actin antibody (A5441) was from Sigma-Aldrich, and monoclonal mouse anticortactin antibody (05-180, clone 4F11) was from Millipore. HRP conjugated- goat anti-Rabbit $\operatorname{IgG}(\mathrm{H}+\mathrm{L})$ (31460), rabbit anti-mouse $\operatorname{IgG}(\mathrm{H}+\mathrm{L})$ (31450) and rabbit anti-goat $\operatorname{IgG}(\mathrm{H}+\mathrm{L})$ (31402) secondary antibodies were purchased from Thermo Fisher Scientific. Thermo Fisher Scientific supplied Alexa conjugated secondary antibodies including Alexa Fluor 350 conjugate Goat anti-Rabbit IgG (H+L) (A-11046), Alexa Fluor 488 conjugate Donkey anti-Goat IgG $(\mathrm{H}+\mathrm{L})(\mathrm{A}-11055)$, Alexa Fluor 546 conjugate Donkey anti-Goat IgG (H+L) (A-11056), Alexa Fluor 555 conjugate Donkey anti-Mouse IgG (H+L) (A-31570), Alexa Fluor 488 conjugate Goat 
anti-Mouse IgG (H+L) (A-11001), Alexa Fluor 555 Phalloidin (A34055) and Alexa Fluor 488 Phalloidin (A12379). Dynasore (D7693) was purchased from Sigma-Aldrich.

\subsection{DNA cloning and mutagenesis}

Gateway cloning technology (Thermo Fisher Scientific) was utilized for DNA cloning. Wild type or $\triangle \mathrm{PRD}$ rat DNM2 was amplified by PCR using the following primers 5'-GGGGACA AGTTTGTACAAAAAAGCAGGCTGCATGGGCAACCGCGGG-3' ${ }^{\prime}$ and GGGGACCACTTTGTACAAGAAAGCTGGGTCGTCGAGCAGGGACGGC-3' for wild type and 5'-GGGGACAAGTTTGTACAAAAAAGCAGGCTGCATGGGCAACCGCGGG-3' and 5'-GGGGACCACTTTGTACAAGAAAGCTGGGTCCGTGGACACGGTGCTGGT-3' for $\triangle \mathrm{PRD}$ from rat wild-type DNM2 cloned in pcDNA4V5/His [15] and subcloned into pDONR201 vector by $\mathrm{BP}$ recombination reaction. The resultant rat DNM2 entry clones were further subcloned into pCi-Ct-EGFP-DEST vector (a generous gift from Dr. Harvey McMahon, LMBMRC) by LR recombination reaction to generate the pCi-rat DNM2-GFP and pCi-rat DNM2 $\triangle$ PRD-GFP constructs.

\subsection{Immunoblotting}

SDS-PAGE samples were prepared from cell extracts of either SV-HUC1 or T24 cells and separated using Mini-PROTEAN System (Bio-Rad). Separated proteins were transferred to nitrocellulose blotting membrane (Amersham Protran Premium $0.45 \mu \mathrm{m}$ NC, GE Healthcare Life Sciences) using Mini Trans-Blot Cell (Bio-Rad). Membranes were blocked in blocking 
buffer (PBS-T with 3\% skimmed milk) for 1 hour at room temperature before incubation with primary antibodies diluted in the blocking buffer (1:1000 for ab52611, sc-6400 and PA1-662; 1:10000 for A5441) overnight at $4^{\circ} \mathrm{C}$ After washing in blocking buffer, membranes were probed with HRP-conjugated secondary antibodies (1:5000 in dilution) for 1 hour at room temperature before signal detection by Amersham ECL Prime Western Blotting Detection Reagent (RPN2232, GE Healthcare) with ChemiDoc Touch Imaging System (Bio-Rad). Protein Expression level was quantified using the Fiji image analysis program [16] .

\subsection{RNAi and transfection}

For RNAi experiment, siGENOME siRNAs for human DNM1 (M-003940-01), human DNM2 (M-004007-03), human DNM3 (M-013931-00) and non-targeting siRNA control (D001206-13-05) were purchased from Dharmacon. T24 cells were transfected with siRNAs using Lipofectamine $^{\mathrm{TM}}$ RNAiMAX Transfection Reagent (Thermo Fisher Scientific) following manufacturer's instruction and resulting phenotypes examined at 72 hours post-transfection.

For the transfection of expression constructs, pCi-rat DNM2-GFP and pCi-rat DNM2 $\triangle \mathrm{PRD}$ GFP were introduced into T24 cells using FuGENE HD Transfection Reagent (Promega) following manufacturer's instruction. Construct transfections were performed at 24 hours postsiRNA transfection and phenotypes were examined at 72 hours after siRNA exposure.

\subsection{Induction of invadopodia formation}


To induce invadopodia formation, gelatin coated coverslips were prepared as previously described [17] with the following modifications. Acid washed coverslips were coated with $50 \mu \mathrm{g} / \mathrm{L}$ poly-L-Lysine, $0.5 \%$ glutaraldehyde, $0.2 \%$ gelatin in order, then aldehyde base was quenched in $5 \mathrm{mg} / \mathrm{mL} \mathrm{NaBH}_{4}$ with gentle washing with PBS between each step. The gelatincoated coverslips were then sterilized in 70\% ethanol, washed in autoclaved PBS and soaked in RPMI-1640 media for 1 hour before using. T24 cells were detached with trypsin-EDTA, seeded onto the prepared gelatin-coated coverslips and incubated in RPMI-1640 media for 3 hours prior to microscopic imaging.

\subsection{Immunofluorescence microscopy}

Cells attached to coverslips were fixed with $4 \%$ paraformaldehyde in PBS for $15 \mathrm{~min}$ at room temperature. After blocking and permeabilization in PBS with 3\% BSA and 0.5\% TX-100 for 1 hour at room temperature, the fixed cells were probed with primary antibodies diluted in antibody buffer (PBS with $1 \%$ BSA and $0.1 \%$ TX-100) overnight at $4^{\circ} \mathrm{C}$. After washing in the antibody buffer, the cells were probed with Alexa-conjugated secondary antibodies diluted in the antibody buffer (1:1000) for 3 hours at room temperature. Treated coverslips were mounted on microscope slides (Matsunami) using Fluoromount/Plus (K048, Diagnostic BioSystems) and images were captured on a fluorescence equipped BX51(Olympus) microscope fitted with cMOS CCD camera (Tucsen MH-15), or captured on a laser scanning confocal microscope (Zeiss LSM-780). Images were analyzed with Fiji and processed using Adobe Photoshop software. 


\subsection{Wound healing assay}

Confluent cells grown in 6-well plates were scratched using a $200 \mu 1$ pipette tip. Recovery processes were captured after 0, 6, 12 and 24 hours using a NEX-5N camera (Sony) attached to CKX41 microscope (Olympus) using a 10× objective lens.

\subsection{Transwell invasion assay}

Transwell invasion assays using Thincert cell culture inserts for 24 well plates (662638, greiner bio-one) were performed following manufacturer's instructions. In brief, Matrigel ${ }^{\mathrm{TM}}$ Matrix Growth Factor Reduced (354230, BD Biosciences) was diluted with Opti-MEM (Thermo Fisher Scientific) to a final concentration of $235 \mu \mathrm{g} / \mathrm{mL}$ and $100 \mu \mathrm{l}$ of the diluted Matrigel added into $8 \mu \mathrm{m}$ pore transwell upper chambers with pre-cooled micropipette tips. The upper chambers in 24 -well plates were then incubated in $37^{\circ} \mathrm{C}$ for 2 hours to solidify the Matrigel. Matrigelcoated upper chambers were then seeded with approx. $2.5 \times 10^{4}$ cells in $200 \mu 1$ Opti-MEM and combined with the lower chambers filled with complete RPMI-1640 medium. For the Dynasore treatment, $80 \mu \mathrm{M}$ (final concentration) of Dynasore was added into upper chambers 6 hours after seeding cells as described previously [18]. 18 hours after seeding, cells were fixed with $100 \%$ methanol and stained with $0.5 \%$ crystal violet after scraping off the cells on the upper surface of the membrane. Images of invasive cells were captured using camera (NEX-5N, Sony) attached to a microscope (CKX41, Olympus) using 10x objective lens. Cells in 10 random fields were counted for quantification. 


\subsection{Statistical analysis}

All the experiments were repeated three times independently. Data were analyzed for statistical significance using GraphPad Prism6 (GraphPad Software). P $<0.05\left(^{*}\right)$ was considered to be statistically significant, $\mathrm{p}<0.01$ and $\mathrm{p}<0.0001$ were shown as $* *$ and $* * * *$ respectively. 


\section{Results}

\subsection{Dynamin2 localizes to invadopodia of bladder cancer cells}

We first examined invadopodia formation in a bladder cancer cell line T24. After seeding T24 cells on gelatin-coated coverslips, discrete foci of cortactin and actin, key components of invadopodia, were formed in the proximity of nuclei (Fig.1A, T24, arrowheads), while these foci were not seen in normal uroepithelial cell line SV-HUC-1 (Fig.1A, SV-HUC-1). Confocal microscopy showed that these foci were protrusions aligned on the ventral surface of T24 (Fig.1B, arrowheads), confirming that these foci are typical invadopodia as described in different type of invasive cancers [5].

We next determined if dynamin isoforms localize to the invadopodia in T24 cells. Immunofluorescence microscopy using specific antibodies against each dynamin isoforms showed dynamin 1 and 3 dispersed in cytoplasm (Fig.1C, left and right rows), but dynamin2 colocalized with cortactin on the invadopodia (Fig.1C, central row, arrowheads). Furthermore, dynamin2 and cortactin also colocalized with MT1-MMP, a key protease required for invasion (Fig.1 D, arrowheads).

We also examined the expression level of dynamin2 in T24 cells. Previous studies showed that cortactin was overexpressed in bladder cancer cells [19]. Immunoblotting analyses showed that dynamin2 (and all other dynamin isoforms) were expressed in both in SV-HUC-1 and T24 (Supplementary Fig.1A). However, unlike cortactin, the level of dynamin2 in T24 was comparable to SV-HUC-1 (Supplementary Fig.1B), suggesting that localization of dynamin2 to 
the invadopodia was not dependent on its expression level. Thus, we confirmed that dynamin 2 was the sole isoform that localized to the invadopodia in bladder cancer cells.

\subsection{Dynamin2 is required for invadopodia formation}

To gain insight into dynamin function at the invadopodia, T24 cells were treated with Dynasore, a GTPase inhibitor for dynamin. Invadopodia formation in T24 cells were not affected by control DMSO treatment (Fig.2 A, DMSO, arrowheads). In contrast, cells treated with Dynasore showed dynamin2, cortactin and actin all dispersed into cytoplasm and did not form invadopodia (Fig.2 A, Dynasore). Since dynamin2 was the sole dynamin isoform localized to the invadopodia, we also inhibited dynamin2 expression by RNAi. Immunoblotting confirmed that dynamin 2 expression could be efficiently reduced after 3 days of RNAi in T24 cells (Fig.2 C). Invadopodia were formed as clustered foci in control RNAi cells (Fig.2 B, control RNAi) and were indistinguishable from untreated or DMSO treated cells. In DNM2 RNAi cells, invadopodia formation was defective and cortactin and actin were dispersed in the cytoplasm phenocopying the Dynasore-treated cells (Fig.2 B, DNM2 RNAi). The proportion of cells with defective invadopodia was $45 \%$ in DNM2 RNAi cells, while none showed similar defects in control RNAi cells or non-treated cells (Fig.2 D), suggesting that the invadopodia defect was specifically not an artefact of RNAi treatment. Quantitative analyses of diameter and abundance of invadopodia revealed that they are $0.62( \pm 0.03) \mu \mathrm{m}$ and $8.4( \pm 0.5)$ per cell in non-treated cells, and $0.58( \pm 0.02) \mu \mathrm{m}$ and $7.9( \pm 0.4)$ per cell in control RNAi cells, while they are $0.35( \pm$ $0.02) \mu \mathrm{m}$ and $38.7( \pm 1.7)$ per cell in dynamin2 RNAi cells, respectively (Fig. 2E and F). Taken 
together, these results suggest that dynamin2 plays essential roles in invadopodia formation, especially in clustering key components of invadopodia such as cortactin and actin.

\subsection{Dynamin2 is required for invasion of bladder cancer cell}

Given that invadopodia formation was impaired by both Dynasore treatment and DNM2 RNAi, we then examined if the invasiveness was affected. We observed Dynasore treatment suppressed T24 cell invasiveness to $27 \%$ of the levels seen in DMSO-treated control cells (Fig.3 A). Similar invasion defects were observed in DNM2 RNAi cells, with $43 \%$ of control RNAi cell levels (Fig.3B). We also examined the implication of these findings using a wound healing assay. Migration of T24 was noticeably reduced after DNM2 RNAi, whereas it was indistinguishable from control RNAi cells after RNAi of either dynamin1 or 3 (Supplementary Fig. 2). Altogether, these results suggest that dynamin2 could play a pivotal role in bladder cancer cell metastasis in vivo.

\subsection{PRD domain of dynamin2 are required for invasiveness of bladder cancer cells}

To gain a mechanistic insight into dynamin2 function in invadopodia, we performed structure-function analyses. The PRD domain of Dynamin2 is known to interact with the SH3 domain of cortactin to form an actin-bundling "ring" complex which stabilizes filopodia in lung cancer cells [14]. To elucidate the requirement of PRD domain in bladder cancer cells, endogenous T24 cell dynamin2 was suppressed by RNAi and then replaced with exogenously expressed siRNA-resistant rat dynamin2 GFP fusions of wild type or PRD domain deleted 
sequence. GFP fusions of wild type rat dynamin2 localized to the invadopodia and rescued defects in invadopodia formation and invasiveness caused by DNM2 RNAi (Fig.4 A and B). In contrast, rat dynamin2-GFP carrying a $\triangle$ PRD mutation did not localize to the invadopodia and failed to rescue the defects of invadopodia formation and invasiveness (Fig.4 A and B). As Dynasore treatment caused invadopodia defects in T24 cells and inhibited invasiveness (Fig.2 and Fig.3), these results suggest that dynamin2 function at the invadopodia requires its GTPase activity and interaction with SH3 domain containing proteins in invasive bladder cancer cells. 


\section{Discussion}

In this study we demonstrate that dynamin2 is essential for invadopodia formation in T24 bladder cancer cells (Fig. 1). Impairment of dynamin2 by either RNAi or drug treatment causes defects in invadopodia formation (Fig. 2) and inhibits cell invasiveness (Fig. 3). We also show that the PRD domain and GTPase activity are both essential for dynamin2 function at the invadopodia (Fig.4), suggesting that dynamin2 may prove a valuable therapeutic target for the treatment of human bladder cancer.

Dynamin2 has been implicated in cell migration and metastatic invasion of several different types of cancers. In lung cancer, dynamin2 stabilizes actin-based structures, lamellipodia and filopodia and promotes cancer cell migration and invasion $[14,18]$. Dynamin2 also promotes lamellipodia formation of pancreatic ductal carcinoma (PDAC), but its regulation is indirect, acting via the Rho-family GTPase Rac1 [20, 21]. Dynamin2 also localizes to the invadopodia in A375MM carcinoma cells and has been implicated in their invasiveness [13]. However, dynamin 2 function in invasive bladder cancer cells remained elusive. In the present study we show for the first time that dynamin2 is also required for the formation and function of invadopodia in bladder cancer cells.

There still some uncertainty about the function of dynamin2 at the invadopodia. Dynamin is an essential component of membrane fission in clathrin-mediated endocytosis [10]. Dynamin also regulates actin dynamics either directly [11] or indirectly via interaction with various actin binding proteins, especially cortactin [12]. Cortactin directly binds to PRD domain of dynamin 2 by its SH3 domain [22] and promotes branched actin assembly via activation of the Arp $2 / 3$ 
complex [23]. The dynamin2-cortactin complex is implicated in multiple actin-driven events including endocytosis, cortical membrane ruffles, podosomes, actin comet tails and invadopodia [10]. Cortactin is overexpressed in many type of cancers and plays essential roles in formation and function of invadopodia [24]. Here we showed that invadopodia formation defects caused by DNM2 RNAi cannot be rescued by expression of $\triangle$ PRD mutants (Fig.4), suggesting that interaction between dynamin 2 and cortactin is essential for invadopodia formation. Interestingly, our prior study demonstrated that the dynamin2-cortactin complex forms "ring-like" complexes which bundles F-actin to stabilize filopodia in lung cancer cells [14]. Given that invadopodia contain similar "core" F-actin bundles, we postulate that dynamin2 and cortactin may form similar "ring" complexes that help to stabilize core F-actin bundles essential for invadopodia formation. Alternatively, dynamin2 may function in transport of MMPs to invadopodia. MMPs are important enzymes secreted by invasive cancer cells that act to degrade ECM to facilitate invasion and metastasis [5]. Multiple MMPs are engaged in invadopodia formation and function, including MT1-MMP [25]. MT1-MMP was shown to be transported to invadopodia contacting site mainly from two sources where dynamin2 plays pivotal roles: clathrin-mediated endocytosis and trans-Golgi network (TGN) [25]. As a recent study demonstrated that dynamin2, cortactin and atypical protein kinase C (aPKC) control trafficking of MT1-MMP to invadopodia in breast cancer cells [26], future studies are needed to unveil the precise roles of dynamin2 at invadopodia in bladder cancer.

In summary, we have identified and characterized the essential function that dynamin2 plays in invadopodia formation and invasiveness of bladder cancer cells. Since bladder cancer 
treatment is frequently ineffective and neoplastic cell invasiveness is difficult to arrest, further studies into the functions of dynamin2 and its potential as a therapeutic target for bladder cancer treatment are warranted. 


\section{Acknowledgements}

This work was supported by JSPS Grant-in-Aid for Scientific Research (No. 26891017), the Okayama Medical foundation and Ryobi Teien Memory Foundation for T.T. This work was also supported by CREST, JST for K.T. Y.Z. was supported by O-NECUS program and M.N. was supported by EPOK program of Okayama University. We also thank Department of Molecular Biology and Biochemistry, Graduate School of Medicine, Dentistry and Pharmaceutical Sciences, Okayama University for the access to Bio-Rad ChemiDoc Touch Imaging system. We thank Dr. Bill Warren for critical reading of the manuscript. Y.Z. thanks Yue Yin for supporting his overseas study.

\section{Conflict of interest}

The authors declare no conflict of interest. 


\section{References}

1. Kaufman, D.S., W.U. Shipley, and A.S. Feldman, Bladder cancer. The Lancet, 2009. 374(9685): p. 239-249.

2. van den Bosch, S. and J. Alfred Witjes, Long-term cancer-specific survival in patients with high-risk, non-muscle-invasive bladder cancer and tumour progression: a systematic review. Eur Urol, 2011. 60(3): p. 493-500.

3. Mitra, A.P., et al., Factors influencing post-recurrence survival in bladder cancer following radical cystectomy. BJU Int, 2012. 109(6): p. 846-54.

4. Nikapota, A.D., et al., Quality of Life after Bladder Cancer: A Prospective Study Comparing Patient-related Outcomes after Radical Surgery or Radical Radiotherapy for Bladder Cancer. Clinical Oncology, 2016. 28(6): p. 373-375.

5. Murphy, D.A. and S.A. Courtneidge, The 'ins' and 'outs' of podosomes and invadopodia: characteristics, formation and function. Nature reviews. Molecular cell biology, 2011. 12(7): p. 413-426.

6. Sutoh, M., et al., Invadopodia formation by bladder tumor cells. Oncol Res, 2010. 19(2): p. 85-92.

7. Revach, O.-Y. and B. Geiger, The interplay between the proteolytic, invasive, and adhesive domains of invadopodia and their roles in cancer invasion. Cell Adhesion \& Migration, 2014. 8(3): p. 215-225.

8. Beaty, B.T. and J. Condeelis, Digging a little deeper: the stages of invadopodium formation and maturation. European journal of cell biology, 2014. 93(0): p. 438-444. 
9. Leong, H.S., et al., Invadopodia are required for cancer cell extravasation and are a therapeutic target for metastasis. Cell Rep, 2014. 8(5): p. 1558-70.

10. Ferguson, S.M. and P. De Camilli, Dynamin, a membrane-remodelling GTPase. Nature reviews Molecular cell biology, 2012. 13(2): p. 75-88.

11. Gu, C., et al., Direct dynamin-actin interactions regulate the actin cytoskeleton. Embo j, 2010. 29(21): p. 3593-606.

12. Mooren, O.L., et al., Dynamin2 GTPase and Cortactin Remodel Actin Filaments. The Journal of Biological Chemistry, 2009. 284(36): p. 23995-24005.

13. Baldassarre, M., et al., Dynamin Participates in Focal Extracellular Matrix Degradation by Invasive Cells. Molecular Biology of the Cell, 2003. 14(3): p. 10741084.

14. Yamada, H., et al., Actin bundling by dynamin 2 and cortactin is implicated in cell migration by stabilizing filopodia in human non-small cell lung carcinoma cells. Int J Oncol, 2016. 49(3): p. 877-86.

15. Tanabe, K. and K. Takei, Dynamic instability of microtubules requires dynamin 2 and is impaired in a Charcot-Marie-Tooth mutant. J Cell Biol, 2009. 185(6): p. 939-48.

16. Schindelin, J., et al., Fiji: an open-source platform for biological-image analysis. Nat Methods, 2012. 9(7): p. 676-82.

17. Artym, V.V., K.M. Yamada, and S.C. Mueller, ECM degradation assays for analyzing local cell invasion. Methods Mol Biol, 2009. 522: p. 211-9.

18. Yamada, H., et al., Dynasore, a dynamin inhibitor, suppresses lamellipodia formation 
and cancer cell invasion by destabilizing actin filaments. Biochemical and Biophysical Research Communications, 2009. 390(4): p. 1142-1148.

19. Guo, G., et al., Whole-genome and whole-exome sequencing of bladder cancer identifies frequent alterations in genes involved in sister chromatid cohesion and segregation. Nat Genet, 2013. 45(12): p. 1459-63.

20. Eppinga, R.D., et al., Increased expression of the large GTPase dynamin 2 potentiates metastatic migration and invasion of pancreatic ductal carcinoma. Oncogene, 2012. 31(10): p. 1228-41.

21. Razidlo, G.L., et al., Dynamin 2 potentiates invasive migration of pancreatic tumor cells through stabilization of the Racl GEF Vav1. Dev Cell, 2013. 24(6): p. 573-85.

22. McNiven, M.A., et al., Regulated Interactions between Dynamin and the Actin-Binding Protein Cortactin Modulate Cell Shape. The Journal of Cell Biology, 2000. 151(1): p. 187-198.

23. Uruno, T., et al., Activation of Arp2/3 complex-mediated actin polymerization by cortactin. Nat Cell Biol, 2001. 3(3): p. 259-66.

24. Kirkbride, K.C., et al., Cortactin: A multifunctional regulator of cellular invasiveness. Cell Adhesion \& Migration, 2011. 5(2): p. 187-198.

25. Poincloux, R., F. Lizárraga, and P. Chavrier, Matrix invasion by tumour cells: a focus on MT1-MMP trafficking to invadopodia. Journal of Cell Science, 2009. 122(17): p. 3015-3024.

26. Rossé, C., et al., Control of MT1-MMP transport by atypical PKC during breast-cancer 
progression. Proceedings of the National Academy of Sciences, 2014. 111(18): p.

E1872-E1879. 


\section{Figure 1. Dynamin 2 localizes to invadopodia in bladder cancer cells}

(A) SV-HUC-1 and T24 cells immunostained to reveal invadopodia markers cortactin (red) and actin (green). The clustered foci of invadopodia were not observed in SV-HUC-1 cells yet are obvious in T24 cells (arrowheads in the magnified images). (B) Confocal microscopic image of T24 cells stained to reveal 3-D localization of cortactin (red) and actin (green) at invadopodia. Note that actin and cortactin colocalize on the protrusions aligned on the ventral cell surface (arrowheads). (C) T24 cells immunostained to reveal localization of endogenous dynamin1, 2 and 3 (green) and cortactin (red). Dynamin2 is localized to the invadopodia (arrowheads in the magnified images), but dynamin1 and 3 are dispersed in cytoplasm. (D) T24 cells immunostained to reveal MT1-MMP (blue), dynamin2 (red) and cortactin (green). Dynamin2 colocalized with MT1-MMP and cortactin on invadopodia (arrowheads). Bars represent $10 \mu \mathrm{m}$ and $1 \mu \mathrm{m}$ in standard and magnified images, respectively. 


\section{Figure 2. Dynamin2 inhibition causes invadopodia formation defects}

(A) T24 cells showing localization of dynamin2 (blue), cortactin (red) and actin (green) after 3 hours treatment with either DMSO or Dynasore. Clustered invadopodia foci in control DMSO treated cells (arrowheads) became dispersed following Dynasore treatment. (B) T24 cells 3 days after RNAi treatment with either control siRNA or DNM2 siRNA showing localization of dynamin2 (blue), cortactin (red) and actin (green). Typically clustered invadopodia foci formed in control RNAi (arrowheads) were dispersed in DNM2 RNAi cells. (C) DNM2 RNAi depletion of dynamin2 was confirmed by immunoblotting using anti-dynamin2 antibody (Dynamin2) relative to the expression level of actin ( $\beta$-actin). (D) Quantification of invadopodia phenotype in T24 cells after 3 days RNAi. In DNM2 RNAi cells, 45\% of cells showed dispersed spots of actin and cortactin, but no similar seen in non-treated or control RNAi cells. More than 200 cells were counted $(\mathrm{N}=3)$ for the quantification. (E) Average number of actin-cortactin dots observed in T24 cells 3 days after RNAi induction. Average number of dots per cells were 8.4 $( \pm 0.5)$ in non-treated cells, $7.9( \pm 0.4)$ in control RNAi cells and $38.7( \pm 1.7)$ in DNM2 RNAi cells. (F) Diameter of actin-cortactin dots in T24 cells after 3 days RNAi. The average diameter of the puncta were $0.62( \pm 0.03) \mu \mathrm{m}$ in non-treated cells, $0.58( \pm 0.02) \mu \mathrm{m}$ in control RNAi cells and $0.35( \pm 0.01) \mu \mathrm{m}$ in DNM2 RNAi cells. Bars represent $10 \mu \mathrm{m}$ in standard images and $1 \mu \mathrm{m}$ in magnified images. 


\section{Figure 3. Invasiveness of bladder cancer requires dynamin 2}

(A) Transwell invasion assay comparing invasiveness of $80 \mu \mathrm{M}$ dynasore treated cells and DMSO control cells. The number of cells invading through the transwell (purple) was decreased to $27 \%$ of DMSO control following Dynasore treatment. (B) Transwell invasion assay comparing invasiveness between DNM2 RNAi cells and control RNAi cells. The number of DNM2 RNAi cells invading through transwell (purple) was decreased to $43 \%$ of control RNAi cells. 


\section{Figure 4. PRD in dynamin 2 is indispensable for invadopodia formation and function}

(A) Dynamin2 depleted T24 cells expressing either wild type or $\triangle \mathrm{PRD}$ rat dynamin2 GFP fusion protein (green) relative to cortactin (red). Invadopodia formation was restored by wildtype rat dynamin2 (left row, arrowheads) but not using the equivalent $\triangle \mathrm{PRD}$ mutant fusion protein (right row). (B) Recovery of invasiveness defects by expression of rat dynamin2. Reduced invasiveness of DNM2 RNAi cells $(19.5 \pm 2.4 \%)$ was partially recovered by expressing wild type rat DNM2 $(37.4 \pm 3.3 \%)$, whereas rat DNM2 $\triangle \mathrm{PRD}$ failed to recover the invasiveness defects $(17.5 \pm 1.1 \%)$. 
Figure 4

A. Merge

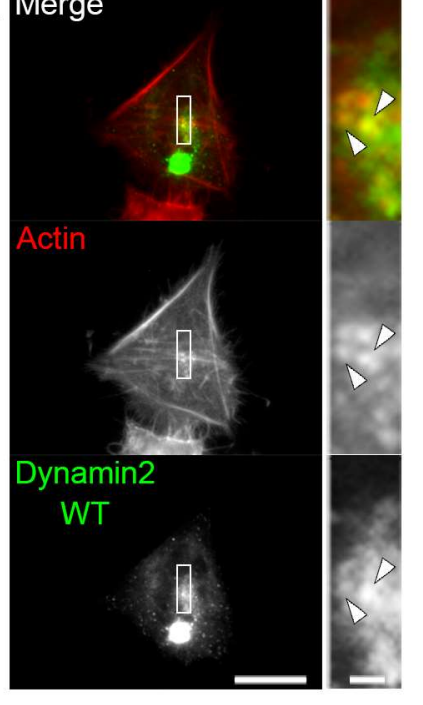

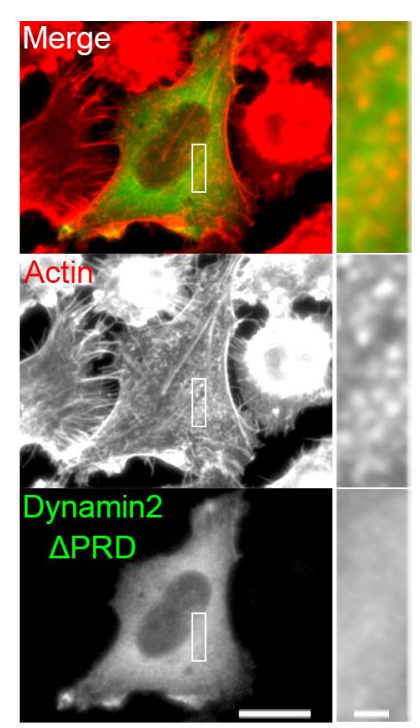

B.

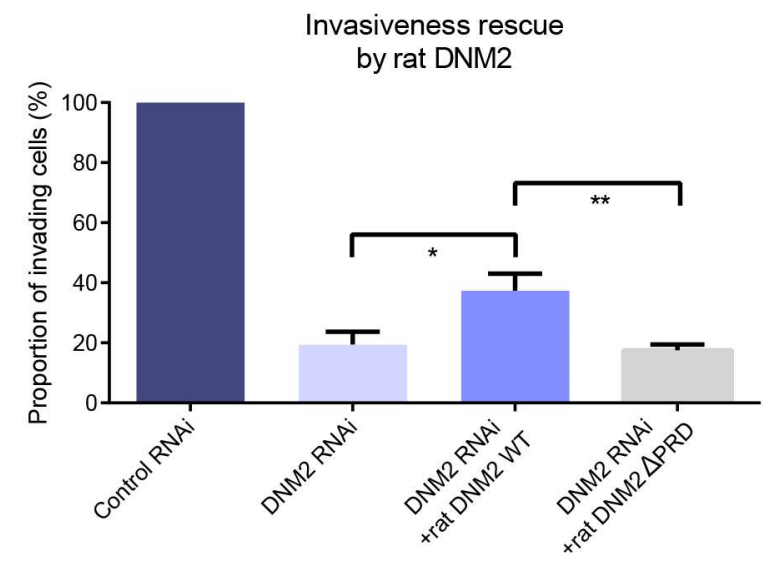


Graphical Abstract

Extracellular Matrix

Actin bundle

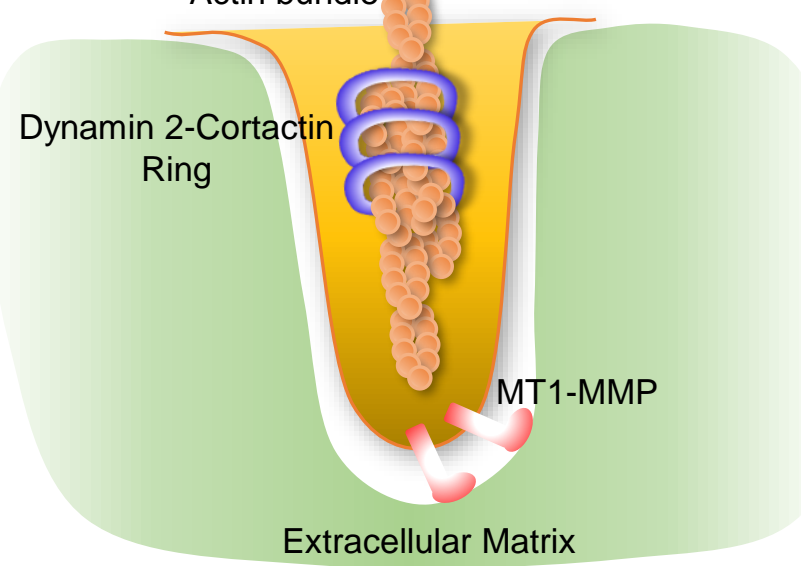

Actin core bundling at invadopodia

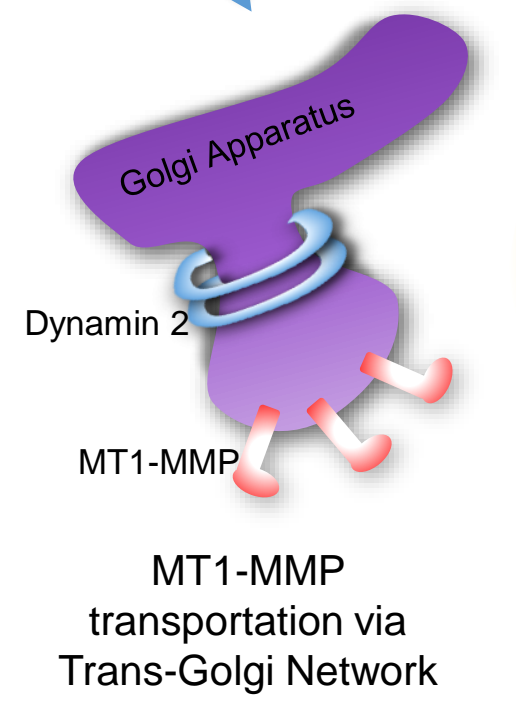

Dynamin 2

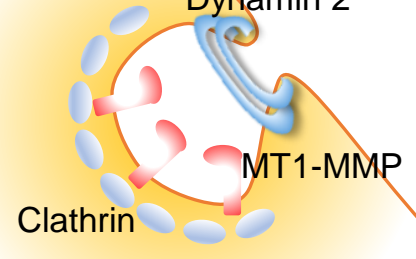

Clathrin-mediated MT1-MMP recycle 